\title{
Circumpapillary and macular vessel density assessment by optical coherence tomography angiography in eyes with temporal hemianopia from chiasmal compression. Correlation with retinal neural and visual field loss
}

\author{
Ana Claudia F. Suzuki ${ }^{1} \cdot$ Leandro C. Zacharias $^{1} \cdot$ Rony C. Preti $^{1} \cdot$ Leonardo P. Cunha $^{1,2} \cdot$ Mário L. R. Monteiro ${ }^{1}$
}

Received: 22 February 2019 / Revised: 12 July 2019 / Accepted: 29 July 2019 / Published online: 18 September 2019

(c) The Author(s), under exclusive licence to The Royal College of Ophthalmologists 2019

\begin{abstract}
Aims To compare the circumpapillary and macular vessel density (cpVD/mVD) of eyes with temporal visual field (VF) defect and band atrophy (BA) of the optic nerve and normal controls using OCTA and to verify the association of VD parameters with circumpapillary retinal nerve fibre layer (cpRNFL) thickness, macular ganglion cell complex (mGCC) thickness and VF loss.

Methods Thirty-three eyes of 26 patients with BA and 42 eyes of 22 age-matched normal controls underwent OCT + OCTA scanning. cpVD and cpRNFL were expressed as average and sector measurements. mVD and mGCC were calculated as averages and in quadrants and hemiretinas. VF loss was estimated using the 24-2 and the 10-2 protocols. Generalized estimated equation models were used for comparisons and area under the receiver operating characteristics (AROC) were calculated.

Results Compared with controls, BA eyes displayed smaller average cpVD and mVD values $(p<0.001$ and AROC $=0.91$ for both). Sectorial measurements were also reduced, especially the nasotemporal sector average $\mathrm{cpVD}(p<0.001$ and AROC $=0.96)$ and the nasal retina mVD measurements $(p<0.001$ and AROC $=0.93)$. cpVD and mVD correlated strongly with corresponding cpRNFL and mGCC thickness measurements in affected regions ( $r$ range: $0.67-0.78$ and $0.56-0.76$, respectively). Similarly, $\mathrm{cpVD}$ and $\mathrm{mVD}$ parameters correlated significantly with corresponding VF loss $(r$ range: $0.45-0.68)$.

Conclusions cpVD and mVD are significantly reduced in BA eyes compared with controls and are strongly correlated with retinal neural and VF loss. cpVD and mVD reduction on OCTA could serve as a surrogate for retinal neural loss in compressive optic neuropathy and might be useful in its management.
\end{abstract}

Supplementary information The online version of this article (https:// doi.org/10.1038/s41433-019-0564-2) contains supplementary material, which is available to authorized users.

Mário L. R. Monteiro

mlrmonteiro@terra.com.br

1 Division of Ophthalmology and the Laboratory of Investigation in Ophthalmology (LIM 33), University of São Paulo, São Paulo, Brazil

2 Department of Ophthalmology, Federal University of Juiz de Fora, Minas Gerais, Brazil

\section{Introduction}

Optical coherence tomography (OCT) is now widely used to detect and quantify retinal axonal loss in glaucoma and a number of other optic pathway diseases [1-4]. More recently, OCT angiography (OCTA), a technology that maps retinal and optic nerve microcirculation, was developed and revolutionized the assessment of retinal vascular diseases [5]. Moreover, studies have shown that OCTA-measured retinal circumpapillary and macular vessel density (cpVD/ $\mathrm{mVD}$ ) can be affected even in patients with nonprimarily vascular optic nerve diseases including glaucoma, inflammatory and hereditary optic neuropathies [6-10]. In many such conditions, however, a contributing vascular component is suspected in the pathogenesis $[8,10,11]$, making it 
difficult to distinguish primary vascular compromise from vascular reduction secondary to retinal neural loss.

Patients with chiasmal lesions may provide an important model to evaluate whether retinal axonal damage is associated with retinal VD reduction. Eyes with temporal hemianopia from chiasmal compression experience preferential damage to axons originating in the nasal hemiretina (which cross in the chiasm) and develop optic nerve head $(\mathrm{ONH})$ fibre loss in a pattern known as band atrophy (BA) of the optic nerve, with axonal loss affecting predominantly the nasal and temporal quadrants of the optic disc [12]. Retinal neural loss tends to affect the nasal and leave the temporal hemiretina relatively preserved $[13,14]$. Because the lesion occurs at the chiasm, there is no indication of primary vascular involvement in the eye.

In this study, we used OCTA to quantitatively assess average and sector $\mathrm{cpVD}$ as well as average, quadrantic and hemiretinal $\mathrm{mVD}$ in patients with BA from chiasmal compression and temporal visual field (VF) defects and compared with controls. We also assessed the correlation of VD with VF defect and retinal axonal loss expressed as thinning of the circumpapillary retinal nerve fibre layer (cpRNFL) and the macular ganglion cell complex (mGCC).

\section{Materials and methods}

This prospective, cross-sectional study included 33 eyes from 26 patients (11 female) with BA and temporal VF defects from previously treated suprasellar tumours (24 pituitary adenoma and 2 craniopharyngioma) and 42 eyes from 22 (13 female) controls. All patients were imaged using MRI scanning to confirm chiasmal compression at the time of diagnosis and to document effective decompression after treatment. Patients had stable VF defects for at least 6 months prior to study entry. Assuming that healthy controls have mVD of $41.2 \pm 3.4 \%$ [7], 24 eyes for each group could permit demonstrating a difference of at least $10 \%$ of mVD with a power of $80 \%$ and a confidence of $95 \%$. Sample size was estimated to compare the two groups, assuming a two-tailed test. We enroled 33 eyes from patients and 42 from controls in order to account for differences in study populations and instrumentation.

Subjects underwent a complete ophthalmologic examination, including VF examination. Among the inclusion criteria were best-corrected VA $\geq 20 / 30$ in the study eye, refractive error within \pm 5 dioptres for the more ametropic meridian and intraocular pressure $<21 \mathrm{mmHg}$. Exclusion criteria were: media opacities, history of other optic neuropathies, retinal diseases, clinical signs of glaucoma, optic disc anomalies, a history of alcohol abuse, tobacco, diabetes mellitus or arterial hypertension treated with more than three antihypertensive medications.
In 19 patients, only one eye met the inclusion criteria while in 7 both eyes were included. The control eyes were from healthy volunteers with normal ophthalmic and VF findings, recruited from among hospital staff. In controls, both eyes were included, except for two subjects (one had cataract; the other corneal opacity in one eye). The study followed the tenets of the Declaration of Helsinki, was approved by the Institutional Review Board Ethics Committee. Informed consent was obtained from all participants. The study was registered at plataformabrasil.saude.gov.br (number 69238217.0.0000.0068).

\section{VF testing}

VF was assessed on standard automated perimetry (SAP) with the 24-2 SITA-Standard strategy (Humphrey Field Analyzer; Carl Zeiss Meditec, Dublin, CA) using Goldmann size III stimulus. After resting for at least $15 \mathrm{~min}$, subjects were also submitted to the SITA 10-2 testing strategy. Reliability criteria were fixation loss $\leq 20 \%$, falsepositive rate $\leq 15 \%$ and false-negative rate $\leq 30 \%$. BA eyes were required to have partial or complete temporal hemianopia and a nasal hemifield within normal limits, defined as the absence of clusters of three or more points with $p<$ $5 \%$ on the pattern deviation plot (24-2 test).

VF sensitivity (VFS) loss were averaged on the total deviation plot in 50 test points of the 24-2 test (excluding the 2 most nasal points, 1 above and 1 below the blind spot) to calculate the mean deviation (MD). VFS loss was also grouped for the nasal hemifield (26 test points), labelled the nasal mean defect (NMD), for the temporal hemifield (24 test points), labelled the temporal mean defect (TMD) and for six VF sectors of the Garway-Heath et al.'s optic disc-VF map [15]. We averaged 21 superior test points corresponding to the inferonasal (IN - sector 2) and inferotemporal (IT - sector 3) disc sectors, 21 inferior test points corresponding to the superotemporal (ST sector 4) and superonasal (SN - sector 5) disc sectors, 6 temporal test points corresponding to the papilomacular bundle and 4 most temporal test points corresponding to the temporal (sector 6) and nasal (sector 1) disc sectors, respectively. VFS loss in the 10-2 test (68 test points) was also averaged globally to calculate the central MD (cMD), in two hemifields (nasal and temporal) and in quadrants. VFS loss was converted from $\mathrm{dB}$ to $1 /$ Lambert linear units by dividing the $\mathrm{dB}$ value by 10 and anti-logging the quotient.

\section{OCT scanning}

Subjects underwent OCT and OCTA scanning using a $1050 \mathrm{~nm}$ swept-source OCT (DRI OCT Triton Plus ${ }^{\oplus}$ V.10.11., Topcon, Japan), with an acquisition speed of 


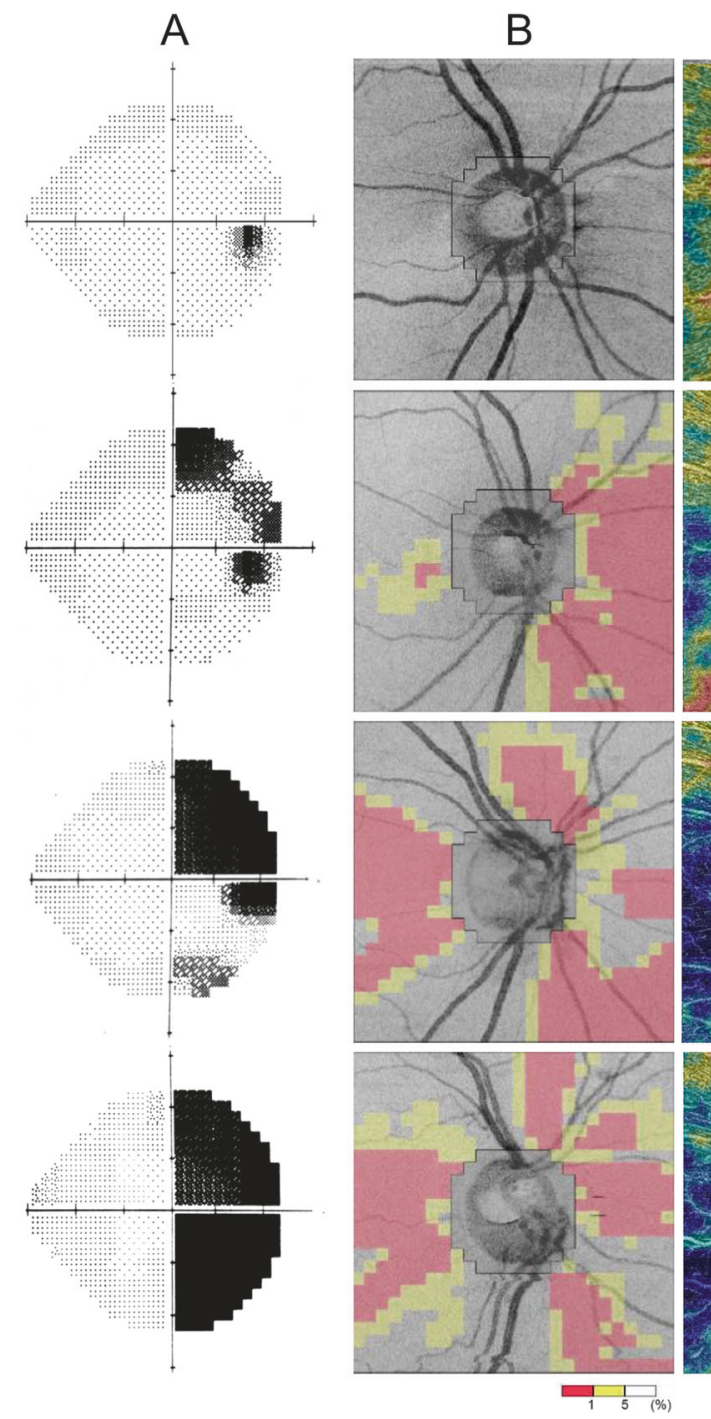

Fig. 1 Samples from a normal subject (first row), two cases with partial temporal visual field defect (second and third rows), and one case with complete temporal visual field defect (bottom row). a grayscale graphic of 24-2 standard automated perimetry (SAP). b significance pattern deviation of circumpapillary RNFL thickness based on the normative range of the DRI OCT Triton Plus ${ }^{\circledR}$ equipment with colour-coded grids (yellow $=$ outside the $95 \%$ normal limit; red

$100,000 \mathrm{~A}$-scans/s. OCT images in a raster pattern covering a $6 \times 6 \mathrm{~mm}$ area of the disc and a $7 \times 7 \mathrm{~mm}$ area of the macula, were obtained. Only well-cantered images with signal intensity $>40$, without motion or blinking artefacts were analysed.

cpRNFL thickness measurements were generated on a circular map $(\varnothing=3.4 \mathrm{~mm})$ drawn around the $\mathrm{ONH}$ when measuring global average thickness $\left(360^{\circ}\right)$ and sector thickness: temporal $\left(310-41^{\circ}\right)$, ST $\left(41-80^{\circ}\right)$, SN $\left(80-120^{\circ}\right)$, nasal $\left(121-230^{\circ}\right)$, IN $\left(231-270^{\circ}\right)$, and $\operatorname{ITm}\left(271-310^{\circ}\right)$, according to the Garway-Heath et al.'s map [15]. In this study, the superior (SN and ST) and inferior (IT and IN) segments were averaged, resulting in four parameters: temporal $\left(90^{\circ}\right)$, superior
C

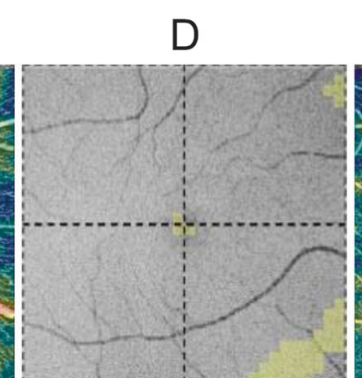

E

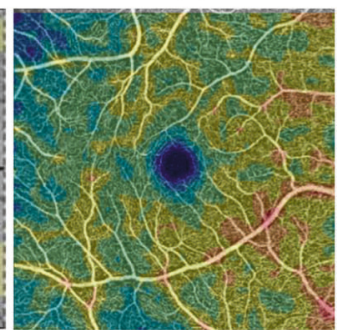

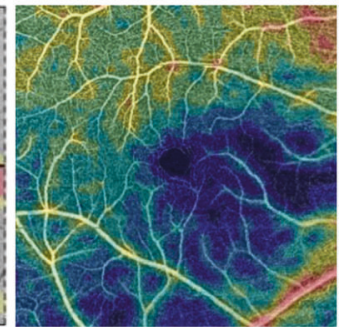

$(1)$
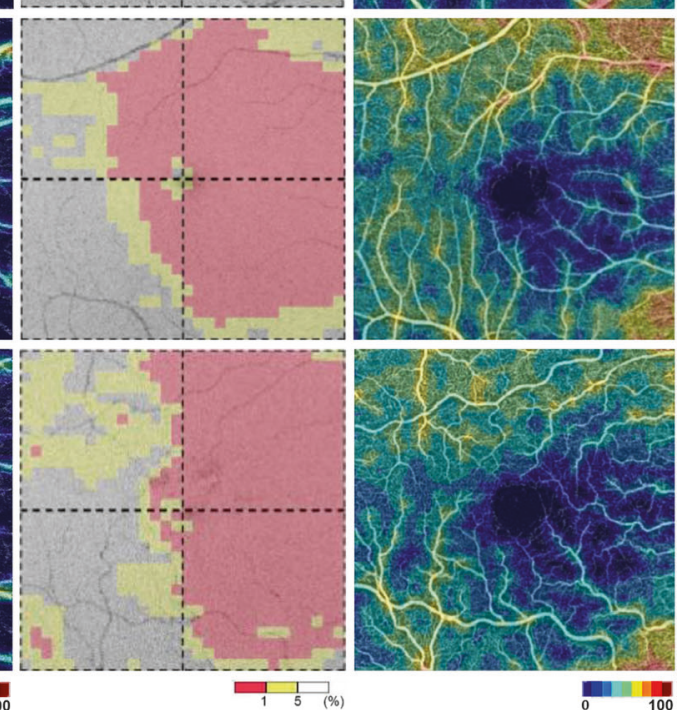

\begin{tabular}{lll}
1 & 1 \\
\hline
\end{tabular}

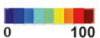

$=$ outside the $99 \%$ normal limit). c pseudo-coloured OCTA circumpapillary density map. d significance pattern of macular ganglion cell complex thickness based on the normative range of the equipment with colour-coded grids (yellow $=$ outside the $95 \%$ normal limit; red $=$ outside the $99 \%$ normal limit). e pseudo-coloured OCTA macular vessel density map

$\left(80^{\circ}\right)$, inferior $\left(80^{\circ}\right)$ and nasal $\left(110^{\circ}\right)$ sectors. We also estimated the superoinferior (SI) sector (average of the superior and the inferior sectors) and the nasotemporal (NT) sector (average of nasal and temporal sectors).

The software automatically segmented the macular thickness measurements, providing the sum of the macular RNFL and the GCL + inner plexiform layer, referred to as the mGCC and registered according to an overlaid OCTgenerated checkerboard (100 checks) covering an area of $6 \times 6 \mathrm{~mm}$ and subsequently averaged in four macular quadrants ( 25 checks). The mGCC global average thickness and the average of the nasal and temporal hemiretinas (50 checks each) were also calculated. 
Table 1 Mean values $( \pm$ standard deviation) circumpapillary and macular vessel density (VD) in 33 eyes with band atrophy (BA) of the optic nerve and 42 healthy control eyes, with area under the receiver operating characteristic curve (AROC)

\begin{tabular}{lllrl}
\hline Parameter & BA eyes $(n=33)$ & Controls $(n=42)$ & $p$-value & AROC (SE) \\
\hline Circumpapillary vessel density $(\%)$ & & & \\
Global average & $34.2 \pm 5.7$ & $43.4 \pm 4.5$ & $<0.001$ & $0.91(0.04)$ \\
Superior sector & $48.8 \pm 9.9$ & $54.4 \pm 7.5$ & 0.011 & $0.71(0.06)$ \\
Temporal sector & $24.2 \pm 8.5$ & $38.0 \pm 8.5$ & $<0.001$ & $0.88(0.04)$ \\
Inferior sector & $47.9 \pm 9.1$ & $56.8 \pm 7.3$ & $<0.001$ & $0.77(0.06)$ \\
Nasal sector & $21.7 \pm 4.0$ & $30.1 \pm 5.9$ & $<0.001$ & $0.89(0.04)$ \\
NT sector average & $22.9 \pm 4.1$ & $33.6 \pm 4.8$ & $<0.001$ & $0.96(0.02)$ \\
SI sector average & $48.4 \pm 8.8$ & $55.6 \pm 6.1$ & $<0.001$ & $0.76(0.06)$ \\
Macular vessel density $(\%)$ & & & & \\
Global average & $21.5 \pm 3.2$ & $26.1 \pm 1.7$ & $<0.001$ & $0.91(0.04)$ \\
SN quadrant & $26.5 \pm 6.5$ & $32.1 \pm 4.8$ & $<0.001$ & $0.78(0.06)$ \\
IN quadrant & $21.9 \pm 6.0$ & $32.4 \pm 3.9$ & $<0.001$ & $0.93(0.03)$ \\
ST quadrant & $21.7 \pm 4.8$ & $20.9 \pm 3.1$ & 0.373 & $0.50(0.07)$ \\
IT quadrant & $15.7 \pm 4.2$ & $18.8 \pm 4.0$ & 0.004 & $0.70(0.06)$ \\
Nasal hemiretina & $24.2 \pm 5.4$ & $32.3 \pm 3.5$ & $<0.001$ & $0.89(0.04)$ \\
Temporal hemiretina & $18.7 \pm 2.8$ & $19.8 \pm 2.4$ & 0.077 & $0.64(0.07)$ \\
\hline
\end{tabular}

$N T$ nasotemporal, $S I$ superoinferior, $S N$ superonasal, $I N$ inferonasal, $S T$ superotemporal, $I T$ inferotemporal, $S E$ standard error

${ }^{\mathrm{a}}$ Generalized estimating equations. Significant values are in italics

\section{OCT angiography}

OCTA data were obtained using the same OCT device. Scans were taken from $4.5 \times 4.5 \mathrm{~mm}$ cubes centred on the disc and $6 \times 6 \mathrm{~mm}$ cubes centred on the fovea (Fig. S1). Enface OCT images should have absent signs of large eye movements (defined as abrupt shift completely disconnecting a large retinal vessel) or black bands (resulting from blinking) and should have uniform signal quality $>40$.

The automated layer segmentation of the equipment's software (IMAGEnet 6 V.1.21.11783) generated different en face slabs. We evaluated cpVD within the cpRNFL in a slab from the inner-limiting membrane (ILM) to the junction of the RNFL/GCL. In the macula, the superficial capillary plexus en face images were generated in a slab from $2.6 \mu \mathrm{m}$ beneath the ILM to $15.6 \mu \mathrm{m}$ beneath the interface of the inner plexiform layer and inner nuclear layer (Fig. S1). Images were reviewed for correct centration and segmentation.

\section{Quantitative VD}

Exported OCTA images were analysed using ImageJ software (National Institute of Health, Bethesda, MD). Images were binarized with intensity thresholding by Otsu's method [16], and VD expressed as a ratio by dividing the total number of pixels coinciding with the vessels by the total number of pixels in the image.

For cpVD evaluation, a circular area $(\emptyset=3.4 \mathrm{~mm})$ centred on the $\mathrm{ONH}$ was delimited. A smaller concentric circle $(\varnothing=1.7 \mathrm{~mm})$ was used to block the data from the disc, limiting the analysis to a halo around the ONH. cpVD was expressed as global average $\left(360^{\circ}\right)$ and four sectors: temporal $90^{\circ}$, superior $80^{\circ}$, nasal $110^{\circ}$ and inferior $80^{\circ}$. We also calculated the NT and SI sector cpVD (average of nasal, temporal, superior and inferior, respectively). These parameters had correspondence with cpRNFL thickness parameters.

Macular VD was expressed as global average, quadrant and hemiretina averages passing through the fovea (Fig. S2). Figure 1 depicts pseudocolored maps representing $\mathrm{cpVD}$ and $\mathrm{mVD}$ in one control and three eyes with BA and different degrees of VF defect.

\section{Data analysis and statistics}

Descriptive statistics included mean values \pm SD. Analysis of histograms and the Kolmogorov-Smirnov test evaluated the normality assumption. The two groups were compared with generalized estimating equation (GEE) models to compensate for intereye correlation from the same subject. Areas under the receiver operating characteristic curves (AROCs) were calculated and compared using DeLong et al.'s method [17].

Correlation of the VD parameters with OCT and VF measurements was analysed with Pearson's correlation test. The level of statistical significance was defined as $p<0.05$. We also estimated the significance at $p<0.01$ and $p<$ 0.001 . Statistical analyses were performed using the software IBM SPSS Statistics V.20. 
Table 2 Mean values ( \pm standard deviation) optical coherence tomography (OCT) circumpapillary retinal nerve fibre layer (cpRNFL) and macular ganglion cell complex (mGCC) (in $\mu \mathrm{m}$ ) in 33 eyes with band atrophy (BA) of the optic nerve and 42 healthy control eyes with areas under the receiver operating characteristic curve (AROC)

\begin{tabular}{llcll}
\hline Parameter & BA eyes $(n=33)$ & Controls $(n=42)$ & $p$-value $^{\mathrm{a}}$ & AROC (SE) \\
\hline cpRNFL thickness $(\mu \mathrm{m})$ & & & & \\
Average & $64.67 \pm 10.53$ & $104.21 \pm 12.09$ & $<0.001$ & $1.00(0.00)$ \\
Superior sector & $90.46 \pm 18.38$ & $127.34 \pm 15.90$ & $<0.001$ & $0.93(0.03)$ \\
Temporal sector & $39.36 \pm 10.87$ & $76.67 \pm 11.12$ & $<0.001$ & $0.99(0.01)$ \\
Inferior sector & $97.83 \pm 16.73$ & $141.89 \pm 22.21$ & $<0.001$ & $0.97(0.02)$ \\
Nasal sector & $42.52 \pm 8.60$ & $82.50 \pm 14.61$ & $<0.001$ & $1.00(0.00)$ \\
NT sector average & $41.09 \pm 7.39$ & $79.88 \pm 9.39$ & $<0.001$ & $1.00(0.00)$ \\
SI sector average & $94.14 \pm 16.58$ & $134.63 \pm 17.00$ & $<0.001$ & $0.97(0.02)$ \\
mGCC thickness $(\mu \mathrm{m})$ & & & & \\
Average & $80.60 \pm 10.17$ & $105.45 \pm 7.57$ & $<0.001$ & $0.99(0.01)$ \\
SN quadrant & $76.64 \pm 11.80$ & $117.36 \pm 9.25$ & $<0.001$ & $0.99(0.01)$ \\
IN quadrant & $82.54 \pm 11.94$ & $119.22 \pm 9.68$ & $<0.001$ & $1.00(0.00)$ \\
ST quadrant & $79.50 \pm 9.82$ & $93.33 \pm 6.85$ & $<0.001$ & $0.83(0.05)$ \\
IT quadrant & $83.74 \pm 9.82$ & $93.33 \pm 6.85$ & $<0.001$ & $0.77(0.06)$ \\
Nasal hemiretina & $79.59 \pm 11.51$ & $118.29 \pm 9.16$ & $<0.001$ & $1.00(0.00)$ \\
Temporal hemiretina & $81.61 \pm 9.68$ & $92.61 \pm 6.78$ & $<0.001$ & $0.81(0.05)$ \\
\hline
\end{tabular}

$R N F L$ retinal nerve fibre layer, $N T$ nasotemporal, $S I$ superoinferior, $S N$ superonasal, $I N$ inferonasal, $S T$ superotemporal, IT inferotemporal, $S E$ standard error

${ }^{a}$ Generalized estimated equations. Significant values are in italics

Table 3 Relationship between circumpapillary vessel density (cpVD), retinal nerve fibre layer (cpRNFL) thickness parameters and visual field (VF) sensitivity loss parameters calculated in the standard automated perimetry (24-2 SITA Standard test)

\begin{tabular}{|c|c|c|c|c|c|c|c|c|c|c|c|c|c|c|}
\hline \multirow{3}{*}{$\begin{array}{l}\text { Parameter } \\
\text { cpVD (\%) }\end{array}$} & \multicolumn{7}{|c|}{ cpRNFL thickness } & \multicolumn{5}{|c|}{ VF sensitivity loss (24-2 test) in $1 / \mathrm{L}$ units } & \multirow{3}{*}{ NMD } & \multirow{3}{*}{ TMD } \\
\hline & \multirow[t]{2}{*}{ GA } & \multirow[t]{2}{*}{ SS } & \multirow[t]{2}{*}{$\mathrm{TS}$} & \multirow[t]{2}{*}{ IS } & \multirow[t]{2}{*}{ NS } & \multirow[t]{2}{*}{ SIS } & \multirow[t]{2}{*}{ NTS } & \multirow[t]{2}{*}{ MD } & \multicolumn{4}{|c|}{ VF sectors [15] } & & \\
\hline & & & & & & & & & 1 & $2+3$ & $4+5$ & 6 & & \\
\hline GA & 0.67 & 0.64 & $\underline{0.69}$ & $\underline{0.62}$ & 0.54 & 0.66 & $\underline{0.65}$ & 0.62 & 0.58 & $\underline{0.55}$ & $\underline{0.61}$ & $\underline{0.56}$ & 0.33 & $\underline{0.61}$ \\
\hline SS & 0.36 & $\underline{0.45}$ & $\underline{0.41}$ & 0.34 & 0.19 & $\underline{0.41}$ & 0.30 & 0.33 & 0.25 & 0.24 & 0.38 & $\mathbf{0 . 3 3}$ & 0.13 & 0.29 \\
\hline TS & $\underline{0.56}$ & $\underline{\mathbf{0 . 5 0}}$ & $\underline{0.73}$ & $\underline{0.52}$ & 0.38 & $\underline{0.53}$ & $\underline{0.56}$ & $\underline{0.51}$ & $\underline{0.52}$ & $\underline{0.44}$ & $\underline{0.49}$ & $\underline{0.48}$ & 0.23 & $\underline{0.51}$ \\
\hline NS & $\underline{0.64}$ & $\underline{0.57}$ & $\underline{0.52}$ & $\underline{0.51}$ & $\underline{0.74}$ & $\underline{0.56}$ & $\underline{0.69}$ & $\underline{0.59}$ & $\underline{0.60}$ & $\underline{0.59}$ & $\underline{\mathbf{0 . 5 3}}$ & $\underline{0.51}$ & 0.32 & $\underline{0.61}$ \\
\hline IS & $\underline{0.48}$ & $\underline{0.48}$ & $\underline{0.42}$ & $\underline{0.52}$ & 0.37 & $\underline{0.52}$ & $\underline{0.42}$ & $\underline{0.48}$ & $\underline{0.41}$ & $\underline{0.42}$ & $\underline{0.49}$ & $\underline{0.40}$ & 0.34 & $\underline{0.46}$ \\
\hline NTS & $\underline{0.73}$ & $\underline{0.64}$ & $\underline{0.78}$ & $\underline{0.63}$ & $\underline{0.65}$ & $\underline{0.66}$ & $\underline{0.75}$ & $\underline{0.66}$ & $\underline{0.67}$ & $\underline{0.61}$ & $\underline{0.61}$ & $\underline{0.61}$ & 0.33 & $\underline{0.68}$ \\
\hline SIS & 0.47 & 0.52 & 0.46 & 0.48 & 0.31 & 0.52 & $\underline{0.40}$ & 0.45 & 0.36 & 0.37 & 0.48 & 0.40 & 0.26 & 0.42 \\
\hline
\end{tabular}

$N=75$. Pearson's correlation coefficients. Italics $=p<0.05$; bold type $=p<0.01$; bold and underlined type $=p<0.001$

GA global average, SS superior sector, TS temporal sector, IS inferior sector, NS nasal sector, SIS superoinferior sector average, NTS nasotemporal sector average, $M D$ mean deviation, $N M D$ nasal mean defect, $T M D$ temporal mean defect

\section{Results}

A total of 33 eyes with BA and 42 control eyes were studied. The average age was $47.8 \pm 12.4$ years (range $21-75$ ) in the BA patients, and $48.4 \pm 14.3$ years (range 24-71) in controls $(p=0.90)$. The mean $\pm \mathrm{SD}$ spherical equivalent was $-0.33 \pm 0.89$ dioptres in the BA group and $+0.14 \pm$ 1.60 in controls $(p=0.22)$. Sixteen eyes presented complete/nearly complete temporal hemianopia, 6 approximately one quadrant VF defect, and 11 defects $<$ one quadrant. MD, TMD and NMD values in BA eyes were $-9.3 \pm 5.1,-19.8 \pm 10.8$ and $-2.1 \pm 1.4 \mathrm{~dB}$, respectively. Corresponding values for normal eyes were $-0.7 \pm 1.2$,
$-0.5 \pm 1.2$ and $-0.9 \pm 1.4 \mathrm{~dB}$. When divided in sectors VF defect was $-21.8 \pm 10.8$ (sector 1), $-9.8 \pm 4.6$ (average sectors 2 and 3), $-7.9 \pm 5.3$ (average sectors 4 and 5) and $-13.5 \pm 9.1 \mathrm{~dB}$ (sector 6 ). Corresponding values for controls were $-0.1 \pm 1.3,-0.7 \pm 1.7,-0.8 \pm 1.2$ and $-0.2 \pm$ $1.3 \mathrm{~dB}$. All VF parameters were significantly smaller in BA patients than controls.

Table 1 and Fig. S3 show the comparisons between VD measurements in both groups. All of the cpVD parameters (except superior sector) were significantly smaller in BA eyes than in controls. The best-performing parameters was the NT sector $\mathrm{cpVD}(\mathrm{AROC}=0.96)$ followed by the global average $\mathrm{cpVD}(\mathrm{AROC}=0.91)$ and the nasal sector $\mathrm{cpVD}$ 
Table 4 Relationship between macular vessel density (mVD) from the superficial capillary plexus (SCP) measurements and macular ganglion cell complex (mGCC) or the central (10-2 test) visual field (VF) loss parameters. Values calculated in quadrants, hemiretinas, hemifields and average measurements

\begin{tabular}{|c|c|c|c|c|c|c|c|c|c|c|c|c|c|c|}
\hline \multirow{3}{*}{$\begin{array}{l}\text { Parameter } \\
\text { mVD (\%) }\end{array}$} & \multicolumn{7}{|c|}{$\mathrm{mGCC}(\mu \mathrm{m})$} & \multicolumn{7}{|c|}{ VF sensitivity loss (10-2 test, 1/L) } \\
\hline & \multirow[t]{2}{*}{ Avg } & \multicolumn{4}{|c|}{ Macular quadrants } & \multirow[t]{2}{*}{ THR } & \multirow[t]{2}{*}{ NHR } & \multirow[t]{2}{*}{ cMD } & \multicolumn{4}{|c|}{ Visual field quadrants } & \multirow[t]{2}{*}{ cNMD } & \multirow[t]{2}{*}{ cTMD } \\
\hline & & SN & IN & ST & IT & & & & $\mathrm{cSN}$ & $\operatorname{cIN}$ & cST & cIT & & \\
\hline Average & $\underline{0.76}$ & $\underline{0.75}$ & $\underline{0.73}$ & $\underline{\mathbf{0 . 7 0}}$ & $\underline{0.64}$ & $\underline{0.68}$ & $\underline{0.75}$ & $\underline{0.68}$ & 0.36 & $\underline{0.45}$ & $\underline{0.66}$ & 0.67 & $\underline{0.44}$ & $\underline{0.68}$ \\
\hline SN quadrant & $\underline{0.57}$ & $\underline{0.56}$ & $\underline{0.54}$ & $\underline{0.58}$ & $\underline{0.47}$ & $\underline{0.54}$ & $\underline{0.55}$ & $\underline{0.46}$ & 0.18 & 0.25 & $\underline{\mathbf{0 . 4 3}}$ & 0.45 & 0.23 & $\underline{0.44}$ \\
\hline IN quadrant & $\underline{0.71}$ & $\underline{0.72}$ & $\underline{0.76}$ & $\underline{0.56}$ & $\underline{0.53}$ & $\underline{0.55}$ & $\underline{0.74}$ & $\underline{0.67}$ & 0.37 & $\underline{0.44}$ & $\underline{0.68}$ & $\underline{0.66}$ & $\underline{0.43}$ & $\underline{0.68}$ \\
\hline ST quadrant & 0.06 & 0.00 & -0.05 & 0.26 & 0.21 & 0.30 & -0.03 & -0.06 & 0.01 & 0.02 & -0.08 & -0.06 & 0.02 & -0.08 \\
\hline IT quadrant & 0.29 & 0.33 & 0.29 & 0.16 & 0.23 & 0.20 & 0.31 & 0.36 & 0.24 & $\underline{0.30}$ & $\underline{0.39}$ & 0.39 & 0.29 & $\underline{0.39}$ \\
\hline THR & 0.28 & 0.27 & 0.20 & 0.33 & 0.35 & 0.34 & 0.24 & 0.26 & 0.21 & 0.27 & 0.25 & 0.28 & 0.25 & 0.27 \\
\hline NHR & $\underline{0.73}$ & $\underline{0.70}$ & 0.74 & 0.64 & $\underline{0.57}$ & $\underline{0.61}$ & $\underline{0.74}$ & $\underline{0.65}$ & 0.32 & 0.39 & 0.64 & 0.64 & 0.38 & $\underline{0.64}$ \\
\hline
\end{tabular}

$N=75$. Pearson's correlation coefficients. Italics $=p<0.05$; bold type $=p<0.01$; bold and underlined type $=p<0.001$

$A v g$ average, $S N$ superonasal, $I N$ inferonasal, $S T$ superotemporal, $I T$ inferotemporal, $T H R$ temporal hemiretina; $N H R$ nasal hemiretina, $c M D$ central mean deviation, $c S N$ central superonasal, $c I N$ central inferonasal, $c S T$ central superotemporal, $c I T$ central inferotemporal, $c N M D$ central nasal mean deviation, $c T M D$ central temporal mean deviation

$(\mathrm{AROC}=0.89)$. Regarding $\mathrm{mVD}$, significant reductions were observed for global average, SN, IN, IT and nasal hemiretina in BA eyes (Table 1). The best-performing $\mathrm{mVD}$ parameter was the IN $\mathrm{mVD}(\mathrm{AROC}=0.93$ ), followed by the average $(\mathrm{AROC}=0.91)$ and the nasal hemiretina $(\mathrm{AROC}=0.89) \mathrm{mVD}$.

Table 2 shows results of OCT cpRNFL and mGCC parameters. The two groups differed significantly in all parameters $(p<0.05, \mathrm{GEE})$. The best-performing cpRNFL parameters were average cpRNFL, NS cpRNFL and NTS cpRNFL $(A R O C=1.00$ for all three). The best-performing mGCC parameter was IN or nasal hemiretina (AROC = 1.00 for both), followed by average or SN mGCC (AROC $=0.99$ for both). The best-performing OCT parameters had significantly greater AROCs than the best-performing OCTA-measured parameters $(p<0.05)$.

Table 3 shows the correlations among cpVD, cpRNFL and VF parameters (24-2 strategy test). The largest correlation coefficients were observed between NT sector cpVD and temporal sector cpRNFL $(r=0.78)$, between NT sector cpVD and NT sector cpRNFL $(r=0.75)$, and between nasal sector cpVD and nasal sector cpRNFL $(r=0.74)$. Regarding the relationship between cpVD measurements and VFS loss, the strongest correlations were observed between NT sector cpVD and three VFS loss parameters: the TMD $(r=$ $0.68)$, VF sector $1(r=0.67)$ and the MD $(r=0.66)$.

Table 4 shows the correlations among mVD, mGCC and 10-2 VF parameters. The strongest correlations were between average $\mathrm{mVD}$ and average $\mathrm{mGCC}$, or between IN $\mathrm{mVD}$ and IN mGCC ( $r=0.76$ for both), followed by the correlation between average $\mathrm{mVD}$ and $\mathrm{SN}$ mGCC or nasal hemiretina mGCC ( $r=0.75$ for both). Highly significant correlations were also found between $\mathrm{mVD}$ and VF parameters based on the 10-2 strategy test. The largest correlation coefficients were between average $\mathrm{mVD}$ and cMD or cTMD ( $r=0.68$ for both) and between IN mVD and cST or cTMD ( $r=0.68$ for both). Figure S4 shows scatterplots of the strongest relationships (OCTA, OCT and VF parameters) encountered.

\section{Discussion}

In the present study, we found that superficial $c p V D$ and mVD parameters effectively differentiate affected eyes with BA and temporal VF defect (stable for at least 6 months) from normal controls. Of the seven cpVD parameters evaluated, all but the superior sector were significantly smaller in BA than in controls. cpVD reduction was most pronounced in the nasal and temporal disc sectors. Similarly, there was significant reduction of nasal hemiretina macular VD parameters in BA eyes (Table 1). These findings are in agreement with the knowledge that eyes with long-standing temporal hemianopia, normal nasal VF and BA of the optic nerve display significant neural loss in the nasal and relative preservation of the temporal hemiretina [18]. Furthermore, the reduction of IT mVD despite the fact that the sample of BA eyes had normal nasal MD, suggests that VD parameters might be more effective than VF parameters at detecting abnormality. The same conclusion is true for the mGCC parameters showing abnormalities in the temporal hemiretina (corresponding to the nasal VF within normal limits) in BA eyes, when compared with controls (Table 2), as previously reported [19-21]. Presumably damage to the uncrossed fibres corresponding to the nasal VF occurred before tumour treatment. An analysis of our OCT and OCTA data shows that the VD parameters matched the abnormalities detected on cpRNFL and mGCC 
with regard to measurements in the nasal hemiretina and the temporal and nasal sectors of the disc (Tables 1,2). Although slightly less efficient than cpRNFL and mGCC parameters, VD measurements displayed high discrimination ability with AROC values as high as 0.96 for NT sector cpVD and 0.93 for IN mVD.

In our study, the correlation coefficients between cpVD and cpRNFL parameters were strong ( $r$ ranging from 0.67 to 0.78 for global average and preferentially affected regions: nasal, temporal and NT sectors), matching the correlation coefficients of earlier studies in glaucoma [6, 9, 22, 23], dominant optic atrophy [8] and neuromyelitis optica [10]. Most previous studies, however, have evaluated only the relationship between global cpVD and cpRNFL; in contrast, we found a strong correlation between the two sets of measurements even when more localized data were analysed, especially the most severely affected areas (nasal sector 0.74, temporal sector 0.73, NT sector 0.78). Regarding the relationship between cpVD and VFS loss, our findings also match the correlation coefficients reported in previous studies that evaluated the relationship between global cpVD and VF MD in different optic nerve diseases [9, 23-25]. In our study, the correlation coefficients between the circumpapillary sectors preferentially affected in BA eyes and VFS loss in corresponding VF areas ranged from 0.48 to 0.68 (Table 3 ), comparable to the relationship between sector cpRNFL thickness parameters and VFS loss in a previous study on a similar set of patients [26].

Our data also shows that BA eyes differed significantly from controls with regard to $\mathrm{mVD}$ values. The discrimination ability was high not only for global average mVD measurement (AROC $=0.91$ ), but also for IN quadrant $(\mathrm{AROC}=0.93)$ and nasal hemiretina $(\mathrm{AROC}=0.89) \mathrm{mVD}$ parameters. Significant differences, although with a slightly worse performance, were also found in the SN (AROC = $0.78)$ and IT $(A R O C=0.70)$ quadrants $\mathrm{mVD}$ parameters (Table 1). Our findings are in agreement with some previous studies in glaucoma [7, 25, 27, 28] and neuromyelitis optica [10] which found reduced average mVD values compared with healthy controls. Nevertheless, other studies have failed to find significantly reduced superficial mVD parameters in patients with glaucoma [23], multiple sclerosis [29] and optic pathway glioma [30]. We believe that some of these discrepancies might be explained by differences in patient selection and scanning protocol. The inclusion in our study of eyes with permanent temporal VF defects and clearly abnormal nasal macular structural parameters provided a robust model capable of evaluating the diagnostic power of $\mathrm{mVD}$ parameters. Furthermore, the use of a sweptsource device and a $6 \times 6 \mathrm{~mm}$ scanning protocol (rather than the more commonly employed $3 \times 3 \mathrm{~mm}$ protocol) likely favoured our results. Since most of the GCL in the macular area is within an $8^{\circ}$ radius circle centred on the fovea [2], most mVD loss secondary to neural degeneration is likely to be beyond the $3 \times 3 \mathrm{~mm}$ central macular area and therefore better assessed with the $6 \times 6 \mathrm{~mm}$ scanning protocol. Our data also shows strong correlations between average/quadrantic/hemifield $\mathrm{mVD}$ and the corresponding $\mathrm{mGCC}$ parameters and VFS loss assessed in directly corresponding regions of the VF using the 10-2 threshold test (Table 4).

While in many optic neuropathies, a vascular mechanism cannot be completely excluded [8, 10, 11], our data of reduced VD parameters in patients with damage from chiasmal compression (i.e., distant from the eye) renders a primary vascular component unlikely and indicates that VD reduction is probably secondary to retinal neural degeneration based on the strong relationships among VD, retinal thickness and VFS loss in directly corresponding areas. The reduction of $\mathrm{cpVD}$ and $\mathrm{mVD}$ may be associated with either capillary drop-out or reduced capillary blood flow possibly related to a reduction in metabolic demand [31, 32]. These observations, however, should be interpreted with caution: OCTA technology is at an early stage, and while its performance was remarkable, it is expected to evolve considerably in the near future. It is therefore not possible to conclude that retinal VD damage occurs only after cpRNFL and mGCC loss or dysfunction. Further developments in OCTA technology and evaluations of VD parameters in compressive chiasmal lesions before and after treatment (and visual recovery) could help to clarify these issues.

Our study was limited by the relatively small sample size and the cross-sectional design, that precluded observation of whether VD reduction might be detected before retinal axonal loss due to dysfunctional (but not atrophic) RCG with reduced energy demands, as suggested in glaucoma [6]. We hope that longitudinal studies of patients with compressive optic pathway diseases will shed more light on the pathophysiology and sequence of changes in VD, axonal and VF parameters.

In conclusion, we found $\mathrm{cpVD}$ and $\mathrm{mVD}$ parameters to be significantly reduced in BA eyes compared with controls, and were strongly correlated with retinal neural and VF loss in patients with chiasmal compression, suggesting that cpVD and mVD reduction could serve as a surrogate for retinal neural loss in compressive optic neuropathy and might be useful in its diagnosis and management. However, further longitudinal studies are necessary to confirm these findings.

\section{Summary}

\section{What was known before}

- Optical coherence tomography angiography shows reduced peripapillary and macular vessel density in a number of anterior optic pathway diseases. Therefore, a 
vascular component is suspected in many such conditions.

\section{What this study adds}

- Eyes with band atrophy of the optic nerve from chiasmal compression differed significantly from controls with regard to circumpapillary and macular vessel density, with reduction significantly correlated with retinal neural loss and visual field loss.

- Circumpapillary vessel density and macular vessel density reduction on OCTA could serve as a surrogate for retinal neural loss in compressive optic neuropathy and might be useful in its management.

Acknowledgements This work is supported by grants from Coordenação de Aperfeiçoamento do Pessoal de Nível Superior (Grant No. 4951-10-07), Brasília, Brazil and and CNPq-Conselho Nacional de Desenvolvimento Científico e Tecnológico (No. 308172/2018-3), Brasília, Brazil. The funding organizations had no role in the design or conduct of this research.

\section{Compliance with ethical standards}

Conflict of interest The authors declare that they have no conflict of interest.

Publisher's note: Springer Nature remains neutral with regard to jurisdictional claims in published maps and institutional affiliations.

\section{References}

1. Hood DC, Kardon RH. A framework for comparing structural and functional measures of glaucomatous damage. Prog Retin Eye Res. 2007;26:688-710. https://doi.org/10.1016/j.preteyeres.2007. 08.001 .

2. Hood DC, Raza AS, de Moraes CG, Liebmann JM, Ritch R. Glaucomatous damage of the macula. Prog Retin Eye Res. 2013;32:1-21. https://doi.org/10.1016/j.preteyeres.2012.08.003.

3. Danesh-Meyer HV, et al. Optical coherence tomography predicts visual outcome for pituitary tumors. J Clin Neurosci. 2015;22:1098-104. https://doi.org/10.1016/j.jocn.2015.02.001.

4. Costello F. Optical coherence tomography in neuroophthalmology. Neurol Clin. 2017;35:153-63. https://doi.org/10. 1016/j.ncl.2016.08.012.

5. Kashani AH, et al. Optical coherence tomography angiography: a comprehensive review of current methods and clinical applications. Prog Retin Eye Res. 2017;60:66-100. https://doi.org/10. 1016/j.preteyeres.2017.07.002.

6. Yarmohammadi A, et al. Relationship between optical coherence tomography angiography vessel density and severity of visual field loss in glaucoma. Ophthalmology. 2016;123:2498-508. https://doi.org/10.1016/j.ophtha.2016.08.041.

7. Akil H, et al. Swept-source OCT angiography imaging of the macular capillary network in glaucoma. Br J Ophthalmol. 2017. https://doi.org/10.1136/bjophthalmol-2016-309816.

8. Balducci N, et al. Optical coherence tomography angiography of the peripapillary retina and optic nerve head in dominant optic atrophy. Mitochondrion. 2017;36:60-5. https://doi.org/10.1016/j. mito.2017.03.002

9. Sakaguchi K, Higashide T, Udagawa S, Ohkubo S, Sugiyama K. Comparison of sectoral structure-function relationships in glaucoma: vessel density versus thickness in the peripapillary retinal nerve fiber layer. Investig Ophthalmol Vis Sci. 2017;58:5251-62. https://doi.org/10.1167/iovs.17-21955.

10. Huang Y, et al. Peripapillary and parafoveal vascular network assessment by optical coherence tomography angiography in aquaporin-4 antibody-positive neuromyelitis optica spectrum disorders. Br J Ophthalmol. 2018. https://doi.org/10.1136/bjophtha lmol-2018-312231.

11. Tobe LA, et al. The role of retrobulbar and retinal circulation on optic nerve head and retinal nerve fibre layer structure in patients with open-angle glaucoma over an 18-month period. Br J Ophthalmol. 2015;99:609-12. https://doi.org/10.1136/bjophthalmol2014-305780.

12. Unsold R, Hoyt WF. Band atrophy of the optic nerve. The histology of temporal hemianopsia. Arch Ophthalmol. 1980; 98:1637-8.

13. Cunha LP, Oyamada MK, Monteiro ML. Pattern electroretinograms for the detection of neural loss in patients with permanent temporal visual field defect from chiasmal compression. Doc Ophthalmol. 2008;117:223-32.

14. Monteiro ML, et al. Evaluation of inner retinal layers in eyes with temporal hemianopic visual loss from chiasmal compression using optical coherence tomography. Investig Ophthalmol Vis Sci. 2014;55:3328-36. https://doi.org/10.1167/iovs.14-14118.

15. Garway-Heath DF, Poinoosawmy D, Fitzke FW, Hitchings RA. Mapping the visual field to the optic disc in normal tension glaucoma eyes. Ophthalmology. 2000;107:1809-15.

16. Schneider CA, Rasband WS, Eliceiri KW. NIH Image to ImageJ: 25 years of image analysis. Nat Methods. 2012;9:671-5.

17. DeLong ER, DeLong DM, Clarke-Pearson DL. Comparing the areas under two or more correlated receiver operating characteristic curves: a nonparametric approach. Biometrics. 1988;44: $837-45$.

18. Akashi A, et al. The detection of macular analysis by SD-OCT for optic chiasmal compression neuropathy and nasotemporal overlap. Investig Ophthalmol Vis Sci. 2014;55:4667-72. https://doi.org/ 10.1167/iovs.14-14766.

19. Tieger MG, et al. Ganglion cell complex loss in chiasmal compression by brain tumors. J Neuroophthalmol. 2017;37:7-12. https://doi.org/10.1097/WNO.0000000000000424.

20. Blanch RJ, Micieli JA, Oyesiku NM, Newman NJ, Biousse V. Optical coherence tomography retinal ganglion cell complex analysis for the detection of early chiasmal compression. Pituitary. 2018;21:515-23. https://doi.org/10.1007/s11102-018-0906-2.

21. Yum HR, Park SH, Park HY, Shin SY. Macular ganglion cell analysis determined by cirrus HD optical coherence tomography for early detecting chiasmal compression. PLoS One. 2016;11: e0153064. https://doi.org/10.1371/journal.pone.0153064.

22. Akil H, Huang AS, Francis BA, Sadda SR, Chopra V. Retinal vessel density from optical coherence tomography angiography to differentiate early glaucoma, pre-perimetric glaucoma and normal eyes. PLoS ONE. 2017;12:e0170476. https://doi.org/10.1371/ journal.pone. 0170476

23. Triolo $G$, et al. Optical coherence tomography angiography macular and peripapillary vessel perfusion density in healthy subjects, glaucoma suspects, and glaucoma patients. Investig Ophthalmol Vis Sci. 2017;58:5713-22. https://doi.org/10.1167/ iovs.17-22865.

24. Yarmohammadi A, et al. Optical coherence tomography angiography vessel density in healthy, glaucoma suspect, and glaucoma eyes. Investig Ophthalmol Vis Sci. 2016;57:451-9. https://doi. org/10.1167/iovs.15-18944. 
25. Chen HS, Liu CH, Wu WC, Tseng HJ, Lee YS. Optical coherence tomography angiography of the superficial microvasculature in the macular and peripapillary areas in glaucomatous and healthy eyes. Investig Ophthalmol Vis Sci. 2017;58:3637-45. https://doi.org/ 10.1167/iovs.17-21846.

26. Monteiro MLR, Costa-Cunha LVF, Cunha LP, Malta RFS. Correlation between macular and retinal nerve fibre layer Fourierdomain OCT measurements and visual field loss in chiasmal compression. Eye. 2010;24:1382-90. https://doi.org/10.1038/eye. 2010.48.

27. Moghimi S, et al. Macular and optic nerve head vessel density and progressive retinal nerve fiber layer loss in glaucoma. Ophthalmology. 2018. https://doi.org/10.1016/j.ophtha.2018.05.006.

28. Wan KH, Lam AKN, Leung CK. Optical coherence tomography angiography compared with optical coherence tomography macular measurements for detection of glaucoma. JAMA
Ophthalmol. 2018;136:866-74. https://doi.org/10.1001/jama ophthalmol.2018.1627.

29. Wang $X$, et al. Optical coherence tomography angiography of optic nerve head and parafovea in multiple sclerosis. Br J Ophthalmol. 2014;98:1368-73. https://doi.org/10.1136/bjophthalmol2013-304547.

30. Parrozzani R, et al. Retinal vascular and neural remodeling secondary to optic nerve axonal degeneration. A study using OCT Angiography. Ophthalmol Retina. 2018;2:827-35. https://doi.org/ 10.1016/j.oret.2017.12.001.

31. Kiryu J, Asrani S, Shahidi M, Mori M, Zeimer R. Local response of the primate retinal microcirculation to increased metabolic demand induced by flicker. Investig Ophthalmol Vis Sci. 1995;36:1240-6.

32. Attwell D, et al. Glial and neuronal control of brain blood flow. Nature. 2010;468:232-43. https://doi.org/10.1038/nature09613. 